\title{
Komme ich da noch raus?
}

\begin{abstract}
Dr. med. M. B., Arzt für Allgemeinmedizin, Lembruch:

Ich habe vor einigen Jahren umfangreich Blutproben für die Polizei abgenommen. Im Rahmen einer Steuerprüfung wurde die Umsatzsteuer für drei Jahre nachträglich erhoben, da ich die Umsatzsteuer-Freigrenze von 17500 Euro überschritten habe. Was ist da schief gelaufen? Was kann ich noch tun?
\end{abstract}

Antwort: Spätestens bei der Bearbeitung der EinnahmenÜberschuss-Rechnung (EÜR) des ersten Jahres mit den hohen Nebeneinnahmen, die darüber hinaus in einem gesonderten Unterkonto erfasst wurden, hätte der Steuerberater den Sachverhalt ansprechen und auf die Umsatzsteuerpflicht hinweisen müssen. Die Umsatzsteuer in Arztpraxen ist seit Jahren ein heißes Eisen für die Finanzbehörde. Insbesondere ein Steuerberater, der Ärzte betreut, muss diese Falle kennen.

Für das erste Jahr, in dem die Grenze von 17500 Euro überschritten wurde, gibt es kaum eine Chance, an der Umsatzsteuernachzahlung vorbeizukommen. Für die weiteren Jahre wird wohl die Haftpflicht des Steuerberaters in Anspruch genommen werden müssen. Um die Situation des ersten Jahrs abzumildern, ist es möglicherweise lohnenswert, die gesamten Aufwendungen im Zusammenhang mit den Blutproben nachträglich durchzuforsten und die Vorsteuer auf diese Aufwendungen zu erfassen. Die Vorsteuer kann dann gegen die Umsatzsteuernachzahlung verrechnet werden. Wenn auf diese Weise vielleicht ein Tausender vermieden werden kann, hat sich die Mühe gelohnt.

Im Prinzip hätte der Steuerprüfer die Umsatzsteuerprüfung bei Überschreiten der Grenze auf weitere Leistungen, die in diesem Augenblick ebenfalls unter die Umsatzsteuerpflicht fallen, ausdehnen können. Seitdem der Europäische Gerichtshof am 14.9.2000 in seinem Urteil mit dem Aktenzeichen Rs C-384/98 entschieden hat, dass ärztliche Leistungen nur dann umsatzsteuerfrei sind, wenn das therapeutische Ziel im Vordergrund steht, hat das Bundesfinanzministeriums dieses Urteil mit Erlass vom 13.2.2001 (IV D 1 - S7170 - 4/01, BStBI. I 2001, 157), umgesetzt. Ein weiteres Schrei- ben des Bundesfinanzministeriums vom 8.11.2001 (IV D 1 - 7170 - 201/01) ergänzt, dass sich die Umsatzsteuerpflicht nun auch auf bestimmte ärztliche Leistungen erstreckt, die im Rahmen einer freiberuflichen Tätigkeit erbracht werden. Die Erstellung eines ärztlichen Gutachtens beispielsweise ist nur dann umsatzsteuerfrei, wenn ein therapeutisches Ziel im Vordergrund steht.

Folglich wäre bei Überschreiten der Freistellungsgrenze die Erstellung von Gutachten für Versicherungsabschlüsse, über die Berufstauglichkeit, die Minderung der Erwerbsfähigkeit umsatzsteuerpflichtig geworden.

Um sich vor diesen Fallen zu schützen, ist es mehr als sinnvoll, die Einnahmen in der Buchführung durch Unterkonten nach möglichst vielen Gesichtspunkten wie Einnahmen durch die KV, Hausarztverträge, Privatpatienten, IGeL sowie Gutachten, Atteste und Leichenschauen zu unterteilen, damit bereits durch die monatliche Auswertung das Erreichen der Freigrenze rechtzeitig erkannt wird.

Ein Steuerberater, der hier nicht mit einer Arztgruppen-spezifischen Kontenführung dem Praxisinhaber zur Seite steht, ist ein „Berater für die Steuer" aber nicht für den Arzt. 